\title{
Dipole Anisotropy in the Lick galaxy catalogue
}

\author{
Manolis Plionis \\ Astronomy Centre, University of Sussex \\ Brighton, BN1 9QH, U.K.
}

The Shane \& Wirtanen (SW) [1] galaxy catalog, as reduced by Seldner et al (SSGP) [2], is used to calculate the dipole vector of the galaxy distribution. The catalogue covers $86 \%$ of the North and $53 \%$ of the South Galactic cap (totally 8.8 steradians) and contains about 810,000 galaxies binned in $10^{\prime} \times 10^{\prime}$ cells with magnitude limit $m_{B} \sim 18.8$. Dipoles have been found in the IRAS and in an optical catalogue based on the ESO, UGC and MCG catalogues, with average depths of $\sim 100 h^{-1}$ and $\sim 50 h^{-1}$ Mpc respectively $[3,4,5,6]$. The direction of these dipoles is consistent with that of the microwave background dipole which means that the structures responsible for the dipole are present within the limits of the shallower catalogue and dominate the large-scale morpology of the galaxy distribution in both catalogues. It can therefore be expected that these structures will be 'washed out' by more distant structures dominating the deeper $\mathrm{SW}$ catalogue. The characteristic depth of the SW catalogue is $360 h^{-1}$ and the median depth of a cluster sample, identified from the SW catalogue by an objective proccedure, is $\sim 180 h^{-1} \mathrm{Mpc}$ [7]. Even if a dipole is found there is no apparent reason for it to point towards the MWB dipole direction since other galaxy fluctuations, comparable in size with those responsible for the MWB dipole, should be present in the SW catalogue if the Cosmological Principle is relevant on scales traced by the catalogue.

There is, though, a significant galaxy number-count anisotropy in the SW catalogue, summarized in Table 1, which adds up to a dipole vector. The dipole vector is calculated by summing moments of the galaxy distribution and using the spherical harmonic expansion of the surface galaxy density to mask the unsurveyed region. Since the SW catalogue has a sharp declination limit, the above calculations are performed in the equatorial coordinate system. Then the corrected, for whole sky coverage, dipole components are rotated to the Galactic coordinate system to model the Galactic obscuration (using a $\operatorname{cosec}(b)$ mask). The dipole found points towards :

$$
\begin{gathered}
l=236^{\circ} .4 \pm 13^{\circ}, b=20^{\circ} .4 \pm 5^{\circ} \\
\text { Amplitude }=6-7 \%
\end{gathered}
$$

which is only $30^{\circ}$ away from the MWB dipole direction, in disagreement with the above speculations. The SW dipole is also $23^{\circ}$ away from both the IRAS and the combined optical dipoles. The errors are estimated by simulating random plate to plate errors and large scale density gradients which are present in the SSGP counts $[8,9]$. Further analysis of the results, of the error calculations and a detail discussion of the findings will appear elsewhere [10]. 


\section{Table 1}

Regions above $|b|=40^{\circ}$. The mean number density per $10^{\prime} \times 10^{\prime}$ cell is listed in the last column.

\begin{tabular}{|l|l|l|l|l|}
\hline Region & Gal.Lat & Declination & Area in $\square^{\circ}$ & $\langle n\rangle$ \\
\hline \hline NGCa & $b>40$ & $\mid$ Dec $\mid<22.5$ & 3535 & 1.51 \\
\hline SGCa & $b<-40$ & $\mid$ Dec $\mid<22.5$ & 3535 & 1.3 \\
\hline NGCb & $b>40$ & $22.5 \leq$ Dec $\leq \mathbf{9 0 . 0}$ & 3767 & 1.33 \\
\hline
\end{tabular}

\section{References}

[1] Shane, C.D. and Wirtanen, C.A. 1967 Publ.LickObs. Vol.XXII, Part 1

[2] Seldner, M., Siebers, B., Groth, E.J., Peebles, P.J.E. 1977 Astron.J. 84, p.249

[3] Yahil, A., Walker, D., Rowan-Robinson, M. 1986 Astrophys.J. 301, L1

[4] Meiksin, A. and Davis, M. 1986 Astron.J. 91, No2, p.191

[5] Lahav, O. 1987 Mon.Not.R.astr.Soc. 225, p.213

[6] Lawrence A., Walker, D. and Rowan-Robinson, M., Leech, K.J., and Penston, M.V. 1986 Mon.Not.R.astr.Soc. 219, p.687

[7] Shectman, S. 1985 Astrophys.J.Suppl. 57, p.77

[8] de Lapparent, V., Kurtz, M.J. and Geller, M.J. 1986 Astrophys.J. 304, p.585

[9] Groth, E.J. and Peebles, P.J.E. 1986b Astrophys.J. 310, p.507

[10] Plionis, M. 1987 in preparation 